Rev Chil Salud Pública 2012;

Vol 16 (1): $16-25$

Artículo original

\section{Satisfacción con la atención médica de pacientes hospitalizados en un servicio clínico docente y en uno no docente}

\section{RESUMEN}

Introducción: En Chile, una proporción importante de hospitales públicos son docente-asistenciales. El objetivo del presente estudio fue determinar y comparar los niveles de satisfacción con la atención médica entre un centro asistencial docente y uno no docente. Material y método: Estudio de corte transversal. Se aplicó un cuestionario para evaluar satisfacción con la atención médica y grado de aceptación hacia estudiantes de Medicina, a pacientes hospitalizados en servicios de urología del Hospital Dr. Gustavo Fricke (no docente) y Hospital Carlos Van Buren (docente), entre septiembre y noviembre de 2009. Se utilizó razón de validez de contenido (CVR) como criterio de inclusión para los diferentes ítems del cuestionario final, que consideró 14 ítems, además de evaluarse su consistencia interna. Resultados: Fueron encuestados 140 pacientes en total, 70 de cada servicio. No hubo diferencia en la satisfacción por servicio (90\% y 88,6\%). Respecto a las variables demográficas, hubo diferencia en edad, proporción de pensionados, y nivel educacional, por servicios. La única variable que mostró asociación con satisfacción fue el sexo, apreciándose en un 95\% de hombres contra un $81,4 \%$ de mujeres $(p=0,01)$. Un $94,3 \%$ de los pacientes aceptó la presencia de los estudiantes en el servicio docente. Discusión: En la muestra estudiada, estar internado en uno u otro centro no afectó la satisfacción de los pacientes. La única variable que se asoció a la satisfacción fue el ser hombre, y la presencia del estudiante fue aceptada por la gran mayoría de pacientes.

Palabras clave: Satisfacción del paciente, atención médica, estudiante de Medicina, hospital docente.

\section{SATISFACTION OF HEALTH CARE IN HOSPITALIZED PATIENTS IN A TEACHING HOSPITAL AND A NON TEACHING HOSPITAL}

\section{ABSTRACT}

Introduction: In Chile, an important proportion of public hospitals are teaching hospitals. The objective of this study was to determine and compare the levels of satisfaction with health care services in one teaching hospital and one non teaching hospital. 
Material and method: Cross-sectional study. A survey was carried out to evaluate satisfaction with health care and the level of acceptance of medical students by hospitalized patients in urology services in Gustavo Frike Hospital (non teaching) and Carlos Van Buren (teaching), between September and November of 2009. Content validity ratios (CVR) was used as inclusion criteria for the 14 different items on the questionnaire, and it was evaluated for internal consistency. Results: 140 patients completed the questionnaire, 70 at each hospital. There was no difference in the level of satisfaction between hospitals $(90 \%$ and $88.6 \%)$. With respect to the demographic variables, between the two hospitals there were differences according to age of patients, proportion of retirees, and level of education. The only variable that was associated with satisfaction was sex, found in $95 \%$ of men and $81.4 \%$ of women $(\mathrm{p}=0.01) .94 .3 \%$ of patients accepted the presence of students at the teaching hospital. Discussion: In this sample, hospitalization in the teaching or the non teaching hospital did not affect patient satisfaction. The only variable that was associated with satisfaction was sex, and the presence of students was accepted by the majority of patients.

Key words: Patient satisfaction, health care, medical students, teaching hospital

\section{INTRODUCCIÓN}

La satisfacción de los pacientes con respecto a la atención en salud se ha transformado en un concepto importante de definir, puesto que constituye una dimensión subjetiva relevante del proceso de calidad de atención que ofrece una institución ${ }^{1}$. Entre las definiciones elaboradas destaca aquella que considera la satisfacción como "la representación de una vivencia subjetiva derivada del cumplimiento o incumplimiento de las expectativas que tiene un sujeto con respecto a algo"2. En términos relacionados con la salud, se referiría a los juicios de valor personales y reacciones subsecuentes a los estímulos que los pacientes, según sus características y experiencias, perciben en los centros de salud ${ }^{3}$.

En términos generales, la satisfacción en salud se refiere a tres aspectos: organizativos (tiempo de espera, ambiente); atención recibida y su repercusión en el estado de salud de la población, y trato recibido durante el proceso de atención por el personal implicado 2 . Por otro lado, desde la perspectiva del paciente, la satisfacción respecto a la atención médica puede dividirse en dos áreas: técnica e interpersonal ${ }^{4}$. A éstas debe agregarse el contexto en el que se desarrolla la atención, donde se considera una gran variedad de factores tales como ambiente, infraestructura, estilo de vida del paciente, sus experiencias previas, expectativas de futuro, sus valores y los de la sociedad, su nivel socioeconó- mico, información recibida por parte del médico y de la enfermera, calidad de la relación médicopaciente, ayuda y actitud general de los médicos del hospital y sus habilidades, experiencia y entrenamiento, entre otros ${ }^{5-9}$. Sin embargo, los factores más influyentes al momento de determinar la satisfacción del paciente son los de tipo perceptivo?.

Existe la creencia de que la presencia del estudiante sería desagradable para el paciente. Sin embargo, varios estudios han concluido que no genera molestias sino más bien beneficios, siendo deseada en algunos $\operatorname{casos}^{10}$, ya que aumenta el tiempo dedicado al paciente, permite solucionar dudas e inquietudes ${ }^{11}$, y favorece el examen físico meticuloso del paciente ${ }^{12}$. La mayoría de los pacientes considera positivo que estudiantes estén envueltos en el cuidado de su salud ${ }^{13,14}$, porque sienten que pueden aportar a su enseñanza ${ }^{15}$, sin embargo les gustaría ser informados previamente ${ }^{16-18}$. Otras publicaciones que se han referido al tema han concluido que los niveles de satisfacción no se ven influidos de manera positiva ni negativa por la presencia del estudiante de medicina ${ }^{17,19,20}$ y otras han demostrado que la percepción de cuidado aumenta cuando hay estudiantes involucrados ${ }^{21}$. Por otro lado, también se ha probado que los pacientes son más reacios a atenderse con estudiantes que pertenecen a cursos menores, o que van a realizar un procedimiento por primera vez 
Tabla 1. Análisis comparativo de variables demográficas entre centros docente y no docente

\begin{tabular}{|c|c|c|c|c|c|}
\hline $\begin{array}{c}\text { Variables } \\
\text { demográficas }\end{array}$ & & $\begin{array}{c}\text { Pacientes } \\
\text { evaluados } \\
(\%)(n=140) \\
\end{array}$ & $\begin{array}{l}\text { Docente } \\
(\%)(n=70)\end{array}$ & $\begin{array}{l}\text { No docente } \\
(\%)(n=70)\end{array}$ & $\begin{array}{c}\text { Valor de } \\
p^{1}\end{array}$ \\
\hline Edad * & $\begin{array}{l}\text { Mediana (RIC) } \\
\text { Adulto } \\
\text { Adulto Mayor }\end{array}$ & $\begin{array}{c}65,1(50-71) \\
62,1 \\
37,9\end{array}$ & $\begin{array}{c}65(54-73) \\
52,9 \\
47,1\end{array}$ & $\begin{array}{c}57,5(43-68) \\
71,4 \\
28,6\end{array}$ & $\begin{array}{c}0,002^{2} \\
0,03\end{array}$ \\
\hline Sexo & $\begin{array}{l}\text { Femenino } \\
\text { Masculino }\end{array}$ & $\begin{array}{l}42,1 \\
57,9\end{array}$ & $\begin{array}{l}34,3 \\
65,7\end{array}$ & $\begin{array}{l}50 \\
50\end{array}$ & $0,087^{3}$ \\
\hline Estado civil & $\begin{array}{l}\text { Soltero/a } \\
\text { Casado/a } \\
\text { Viudo/a } \\
\text { Separado/a } \\
\text { Conviviente }\end{array}$ & $\begin{array}{c}20 \\
56,4 \\
15,7 \\
5 \\
2,9\end{array}$ & $\begin{array}{c}18,6 \\
54,3 \\
20 \\
4,3 \\
2,8\end{array}$ & $\begin{array}{l}21,4 \\
58,6 \\
11,4 \\
5,7 \\
2,9\end{array}$ & $\begin{array}{l}0,67 \\
0,60 \\
0,16 \\
0,69 \\
1,00\end{array}$ \\
\hline Nivel educacional ** & $\begin{array}{l}\text { Secundaria } \\
\text { Superior }\end{array}$ & $\begin{array}{l}84,3 \\
15,7\end{array}$ & $\begin{array}{l}91,4 \\
8,6\end{array}$ & $\begin{array}{l}77,1 \\
22,9\end{array}$ & $0,03^{3}$ \\
\hline Ocupación & $\begin{array}{l}\text { Estudiante } \\
\text { Trabajador } \\
\text { Cesante } \\
\text { Dueña de Casa } \\
\text { Pensionado }\end{array}$ & $\begin{array}{c}0,7 \\
40,7 \\
3,6 \\
22,9 \\
32,1\end{array}$ & $\begin{array}{c}1,4 \\
34,3 \\
2,9 \\
21,4 \\
40\end{array}$ & $\begin{array}{c}0 \\
47,1 \\
4,3 \\
24,3 \\
24,3\end{array}$ & $\begin{array}{l}1,00 \\
0,12 \\
0,64 \\
0,68 \\
0,04\end{array}$ \\
\hline $\begin{array}{l}\text { Experiencias previas } \\
\text { en otros servicios }\end{array}$ & $\begin{array}{l}\text { No } \\
\text { Sí }\end{array}$ & $\begin{array}{l}48,6 \\
51,4\end{array}$ & $\begin{array}{l}51,4 \\
48,6\end{array}$ & $\begin{array}{l}45,7 \\
54,3\end{array}$ & $0,61^{3}$ \\
\hline $\begin{array}{l}\text { Hospitalizaciones } \\
\text { previas }\end{array}$ & $\begin{array}{l}\text { Una vez } \\
\text { Dos veces } \\
\text { Tres o más } \\
\text { veces }\end{array}$ & $\begin{array}{l}61,4 \\
19,3 \\
19,3\end{array}$ & $\begin{array}{c}61,4 \\
20 \\
18,6\end{array}$ & $\begin{array}{c}61,4 \\
18,6 \\
20\end{array}$ & $\begin{array}{l}1,00 \\
0,83 \\
0,83\end{array}$ \\
\hline Tiempo de estadía & $\begin{array}{l}2 \text { a } 4 \text { días } \\
5 \text { a } 10 \text { días } \\
11 \text { o más días }\end{array}$ & $\begin{array}{c}37,1 \\
42,1 \\
20\end{array}$ & $\begin{array}{l}37,1 \\
45,7 \\
17,2\end{array}$ & $\begin{array}{l}38,6 \\
38,6 \\
22,8\end{array}$ & $\begin{array}{l}0,86 \\
0,39 \\
0,39\end{array}$ \\
\hline
\end{tabular}

${ }^{*}$ )Adulto: 18-65 años / Adulto Mayor: mayor de 65 años.

${ }^{* *}$ Secundaria: Hasta secundaria completa, o menos / Superior: Superior técnica o universitaria, completa o incompleta.

RIC: Rango Intercuartil

${ }^{1}$ Test Z

${ }^{2}$ Mann Whitney

${ }^{3}$ Test exacto de Fisher

en ellos 22,23 , o ante situaciones que involucren problemas emocionales, sexuales o exámenes urogenitales ${ }^{24}$.

Los hospitales públicos docentes juegan un rol central en la educación médica y representan la convergencia de la misión de todo sistema de salud de asegurar un excelente cuidado de la salud de su población y la misión de los centros formadores de médicos de entrenar a sus estu- diantes para que sean los mejores médicos ${ }^{25}$.

Pese a que los sectores de educación y salud parecen ser independientes, al momento de analizar la formación de futuros profesionales de salud terminan relacionándose a tal punto que exigen la existencia de una normativa clara respecto a cómo y dónde dichos profesionales pondrán en práctica las enseñanzas que sus centros formadores les han entregado. En Chile, 
se trata de un tema relevante en discusión durante los últimos años que ha llevado a plantear reformas a las normas que rigen los convenios docente-asistenciales desde el siglo XIX. Pese a controversias como la "no cohabitación" y la exclusividad en el uso de un campo clínico por una institución determinada, además de la compensación económica que algunos servicios de salud exigen por su utilización, se acepta que para la docencia en el área de salud es imprescindible contar con un centro clínico en el que reside la base del aprendizaje formal e informal de los estudiantes ${ }^{26}$. Si se considera, además, la fuerza que ha tomado el nuevo paradigma de la medicina que refuerza la importancia de la interacción médico-paciente y de una relación horizontal de ayuda en desmedro del antiguo autoritarismo y relación vertical de dependencia, se vuelve interesante analizar cuál es la opinión y aceptación de los pacientes hacia la presencia de estudiantes de medicina involucrados en su atención durante su estadía hospitalaria y si la presencia de estos mejora o no su satisfacción en relación a centros donde han sido atendidos previamente sin estudiantes.

\section{MATERIAL Y MÉTODO}

Se realizó un estudio de corte transversal que incluyó pacientes hospitalizados en los servicios de Urología del Hospital Dr. Gustavo Fricke de Viña del Mar (HGF) y del Hospital Carlos Van Buren de Valparaíso (HCVB), entre los meses de septiembre y noviembre de 2009. El protocolo del estudio fue aprobado por el comité de ética de la Universidad de Valparaíso. Cada paciente fue informado de la realización de la encuesta y aceptó participar en esta mediante firma de un consentimiento informado.

Fueron incluidos pacientes mayores de 18 años, con estadía de al menos 2 días en sala, y que hubieran tenido contacto con estudiantes de Medicina durante esta. Se excluyeron pacientes alterados de conciencia, con facultades mentales perturbadas, o incapaces de contestar el cuestionario. Se consideró como centro no docente al HGF, puesto que no contaba con la presencia de estudiantes ni internos de Medicina, ni de médicos en especialización (residentes), y como centro docente al HCVB, ya que sí contaba con todos ellos. Para los fines de este estudio se consideró como "estudiante" a todo alumno o médico en formación (alumnos, internos, y residentes), ya que en todos esos casos ellos realizan la atención a los pacientes supervisados por alguien de mayor jerarquía, por lo que son reconocibles como personas en aprendizaje por los pacientes; sin embargo, la mayoría de los estudiantes correspondió a internos de $6^{\circ}$ y $7^{\circ}$ año.

Fueron evaluados todos los pacientes que accedieron a participar de la investigación durante visitas tres veces por semana durante tres meses. Se utilizó un muestreo por conveniencia de todos los pacientes que se alcanzaran a reclutar durante los meses del estudio. El universo del cual provenían los pacientes correspondía a beneficiarios de los Hospitales incluidos en el estudio, los que eran mayoritariamente beneficiarios del Servicio de Salud Viña del Mar-Quillota (HGF) y del Servicio de Salud ValparaísoSan Antonio (HCVB).

Como instrumento de recolección se utilizó un cuestionario con preguntas de selección múltiple, confeccionado para medir los niveles de satisfacción usuaria respecto a la atención médica y los niveles de aceptación de los pacientes hacia los estudiantes de Medicina. Dicho cuestionario constó de dos secciones: una dirigida a evaluar la satisfacción, compuesta por cuatro bloques de preguntas: "aspectos generales", "relación médico-paciente", "comunicación e información", y "percepción de competencias técnico-profesionales del médico"; y la otra, que sólo se aplicó en el centro docente, dirigida a evaluar el grado de aceptación del estudiante. Previo a su aplicación, el contenido del cuestionario fue sometido a un proceso de validación de contenido, mediante juicio de 9 expertos y posterior cálculo de la "razón de validez de contenido" (CVR). Se formuló inicialmente un total de 81 preguntas, de las cuales 14 conformaron el cuestionario definitivo.

Para analizar las variables del cuestionario de satisfacción se utilizó una escala tipo Likert modificada para puntuar las respuestas que iban desde "Muy insatisfecho" a "Muy satisfecho" con puntajes desde (-2) a (+2). En los resultados se muestran las opciones extremas agrupadas, de manera de juntar las respuestas (-2) y $(-1)$, y $(+1)$ y $(+2)$, y dejar un tercer grupo con los "In- 
Tabla 2. Análisis comparativo de respuestas a cuestionario de satisfacción usuaria con la atención médica en centros docente y no docente.

\begin{tabular}{|c|c|c|c|c|}
\hline \multicolumn{2}{|l|}{ Variable respuesta cuestionario } & \multirow{2}{*}{$\begin{array}{c}\begin{array}{c}\text { Docente }(\%) \\
(n=70)\end{array} \\
8,6\end{array}$} & \multirow{2}{*}{$\begin{array}{c}\begin{array}{c}\text { No docente } \\
(\%)(\mathrm{n}=70)\end{array} \\
7,1\end{array}$} & \multirow{2}{*}{$\frac{\text { Valor de } \mathbf{p} \mathbf{1}}{0,74}$} \\
\hline & No & & & \\
\hline Recomendación del servicio & Ind. & 1,4 & 1,4 & 1,00 \\
\hline & Sí & 90 & 91,5 & 0,76 \\
\hline \multirow{3}{*}{ Calidad de la atención } & $\begin{array}{l}\text { M. Insat. o } \\
\text { Insat. }\end{array}$ & 5,7 & 5,7 & 1,00 \\
\hline & $\begin{array}{l}\text { Ind. } \\
\text { Sat. o }\end{array}$ & 1,4 & 2,9 & 0,54 \\
\hline & M. Sat. & 92,9 & 91,4 & 0,74 \\
\hline \multirow{3}{*}{ Relación médico-paciente } & $\begin{array}{l}\text { M. Insat. o } \\
\text { Insat. }\end{array}$ & 11,4 & 8,6 & 0,58 \\
\hline & $\begin{array}{l}\text { Ind. } \\
\text { Sat. o }\end{array}$ & 0 & 5,7 & 0,04 \\
\hline & M. Sat. & 88,6 & 85,7 & 0,60 \\
\hline \multirow{3}{*}{ Confidencialidad del médico } & $\begin{array}{l}\text { M. Insat. o } \\
\text { Insat. }\end{array}$ & 5,7 & 4,3 & 0,70 \\
\hline & $\begin{array}{l}\text { Ind. } \\
\text { Sat. o }\end{array}$ & 2,9 & 5,7 & 0,41 \\
\hline & M. Sat. & 91,4 & 90 & 0,77 \\
\hline \multirow{3}{*}{ Respeto a miedos del paciente } & $\begin{array}{l}\text { M. Insat. o } \\
\text { Insat. }\end{array}$ & 2,9 & 5,7 & 0,41 \\
\hline & $\begin{array}{l}\text { Ind. } \\
\text { Sat. o }\end{array}$ & 1,4 & 5,7 & 0,16 \\
\hline & M. Sat. & 95,7 & 88,6 & 0,11 \\
\hline \multirow{3}{*}{$\begin{array}{l}\text { Claridad en explicación de } \\
\text { enfermedad }\end{array}$} & $\begin{array}{l}\text { M. Insat. o } \\
\text { Insat. }\end{array}$ & 7,3 & 11,4 & 0,40 \\
\hline & $\begin{array}{l}\text { Ind. } \\
\text { Sat o }\end{array}$ & 1,43 & 5,7 & 0,16 \\
\hline & M. Sat. & 91,4 & 82,9 & 0,13 \\
\hline \multirow{3}{*}{ Información sobre tratamiento } & $\begin{array}{l}\text { M. Insat. o } \\
\text { Insat. }\end{array}$ & 11,4 & 8,6 & 0,58 \\
\hline & $\begin{array}{l}\text { Ind. } \\
\text { Sat. o }\end{array}$ & 7,2 & 5,7 & 0,71 \\
\hline & M. Sat. & 81,4 & 85,7 & 0,49 \\
\hline \multirow{3}{*}{ Seguridad del médico } & $\begin{array}{l}\text { M. Insat. o } \\
\text { Insat. }\end{array}$ & 2,8 & 4,3 & 0,63 \\
\hline & $\begin{array}{l}\text { Ind. } \\
\text { Sat. o }\end{array}$ & 2,9 & 1,4 & 0,54 \\
\hline & M. Sat. & 94,3 & 94,3 & 1,00 \\
\hline Puntaje satisfacción operados* & $\begin{array}{l}\text { Mediana } \\
\text { (RIC) }\end{array}$ & $18(14-20)$ & $17(12-20)$ & $0,79^{2}$ \\
\hline $\begin{array}{l}\text { Puntaje satisfacción no } \\
\text { operados** }^{\star *}\end{array}$ & $\begin{array}{l}\text { Mediana } \\
(\mathrm{RIC})\end{array}$ & $13(7-16)$ & $15(9,5-17)$ & $0,40^{2}$ \\
\hline
\end{tabular}

*Puntaje máximo: +21 puntos / Puntaje mínimo: -21 puntos

**Puntaje máximo: +17 puntos / Puntaje mínimo: -17 puntos

*M. Insat: Muy insatisfecho / Insat.: insatisfecho / Ind.: indiferente / Sat.: satisfecho / M. Sat: Muy satisfecho

${ }^{1}$ Test Z

${ }^{2}$ Mann Whitney RIC: Rango Intercuartil 
diferente" (0). En la sección dirigida a evaluar el grado de aceptación hacia el estudiante, las respuestas posibles fueron "Le agrada", "Le da igual" y "Le desagrada" con puntajes desde (-1) a (+1). La "Satisfacción" fue operacionalizada como 6 o más puntos en pacientes no operados, y 7 o más puntos en pacientes operados. Esto se determinó de acuerdo a la opinión de los expertos, estableciendo el punto de corte en puntajes finales equivalentes a haber contestado "Satisfecho" en la mitad más 1 de las preguntas e "Indiferente" en el resto. Respecto al nivel de aceptación hacia estudiantes, el punto de corte para calificar que a un paciente "Le agrada" la presencia del estudiante, fue de 20 más puntos, equivaliendo esto a dos respuestas "Le agrada" y una "Indiferente", de un total de tres preguntas. Para determinar la aceptación hacia el estudiante, el valor de corte fue 1 punto o más, lo que equivale a un "Le agrada" y dos "Le da igual", o bien, dos "Le agrada" y un "Le desagrada".

Las respuestas del cuestionario fueron sometidas a análisis estadístico con Stata 10. Se usaron frecuencias absolutas y relativas para variables cualitativas, y medianas y rango intercuartil (RIC) para cuantitativas. Se usó test Z para comparación de proporciones, test exacto de Fisher para asociación entre variables cualitativas, y Mann Whitney para comparación de medianas. Se realizó un análisis de asociación exploratoria entre variables demográficas y nivel de satisfacción para todos los pacientes, y posteriormente, de acuerdo al tipo de centro. Para el análisis del instrumento, se aceptaron ítems con una CVR $\geq 0,75$ (Lawshe, 1975). Para evaluar la consistencia interna de los ítems se usó alfa de Cronbach, considerando como relevantes valores de alfa iguales o mayores a 0,7. Para todos los análisis se consideró $\mathrm{a}=0,05$.

\section{RESULTADOS.}

Se encuestó un total de 140 pacientes, correspondientes a 70 del HCVB (de 120 hospitalizados, 58,3\% del total), y 70 del HGF (de 136 hospitalizados, $51,47 \%$ del total). Sin embargo, estos correspondieron a más del $95 \%$ de los pacientes urológicos de cada centro, debido a que gran parte de los pacientes hospitalizados eran de otras especialidades, y estaban en el servicio de urología debido al sistema de gestión en red.

La mediana (RIC) de edad fue 65,1 (50-71) años de edad, correspondiendo a $57,9 \%$ hombres y $42,1 \%$ mujeres. En el centro docente la

Tabla 3. Descripción del grado de aceptación hacia estudiantes de medicina en el centro docente.

\begin{tabular}{|c|c|c|c|c|}
\hline $\begin{array}{l}\text { Variables respuesta } \\
\text { cuestionario estudiantes }\end{array}$ & & $\begin{array}{c}\begin{array}{c}\text { Frecuencia } \\
(n=70)\end{array} \\
\end{array}$ & $\begin{array}{l}\text { Porcentaje } \\
(\%)(n=70)\end{array}$ & $\begin{array}{c}\text { Mediana } \\
(\mathrm{RIC})(\mathrm{n}=70) \\
\end{array}$ \\
\hline $\begin{array}{l}\text { Participar en la enseñanza } \\
\text { del estudiante de Medicina }\end{array}$ & $\begin{array}{l}\text { Le desagrada } \\
\text { Le da igual } \\
\text { Le agrada }\end{array}$ & $\begin{array}{c}2 \\
9 \\
59\end{array}$ & $\begin{array}{c}2,8 \\
12,8 \\
84,4\end{array}$ & \\
\hline $\begin{array}{l}\text { Ser examinado en presencia } \\
\text { de un estudiante de Medicina }\end{array}$ & $\begin{array}{l}\text { Le desagrada } \\
\text { Le da igual } \\
\text { Le agrada }\end{array}$ & $\begin{array}{c}2 \\
23 \\
45\end{array}$ & $\begin{array}{c}2,8 \\
32,8 \\
64,4\end{array}$ & \\
\hline $\begin{array}{l}\text { Respeto a la confidencialidad } \\
\text { del paciente }\end{array}$ & $\begin{array}{l}\text { No } \\
\text { Le da igual } \\
\text { Sí }\end{array}$ & $\begin{array}{c}- \\
4 \\
66\end{array}$ & $\begin{array}{c}- \\
5,7 \\
94,3\end{array}$ & \\
\hline $\begin{array}{l}\text { Puntaje de aceptación hacia } \\
\text { el estudiante de Medicina* }\end{array}$ & & & & $2,38+0,82$ \\
\hline $\begin{array}{l}\text { Aceptación hacia el estudiante } \\
\text { de Medicina }\end{array}$ & $\begin{array}{l}\text { No acepta } \\
\text { Sí acepta }\end{array}$ & & $\begin{array}{l}5,7 \\
94,3\end{array}$ & \\
\hline
\end{tabular}

* Puntaje Máximo: +3 / Puntaje Mínimo: -3

RIC: Rango Intercuartil 
mediana fue 65 (54-73) años, y en el no docente 57,5 (43-68) $(p=0,002)$. Hubo diferencia significativa en el grupo de adultos mayores de 65 años, correspondiendo a un $47,1 \%$ en el centro docente y a $28,6 \%$ en el no docente $(p=0,02)$. Respecto al nivel educacional, en el centro docente hubo un $8,6 \%$ de personas que habían cursado al menos algún estudio superior, mientras que en el no docente fue de $22,9 \%(p=0,03)$. En el grupo de pensionados se encontró una diferencia de $15,7 \%$ entre ambos centros, siendo $40 \%$ en el centro docente versus $24,2 \%$ en el no docente $(p=0,04)$. No hubo diferencia por sexo ni en las otras variables estudiadas entre los dos grupos (Tabla 1).

Respecto al cuestionario, todas las preguntas incluidas presentaron una CVR $\geq 0,75$, y las dimensiones un alfa de Cronbach $>0,7$. Al analizar las variables, se observó que no existió diferencia significativa en ninguna de las dimensiones estudiadas entre ambos servicios, al considerar las respuestas extremas (es decir, los "Insatisfechos" y "Muy insatisfechos", o los "Satisfechos" y "Muy satisfechos"). Hay que resaltar que en prácticamente todas las dimensiones evaluadas hubo más de un $85 \%$ de respuestas "Satisfecho" o "Muy satisfecho" (Tabla 2). Se aprecia la comparación gráfica de la dimensión "Calidad de la atención" entre el servicio docente y el no docente (Figura 1). No se evidenció asociación significativa entre el nivel de satisfacción de los pacientes en relación al centro hospitalario en el que se encontraban hospitalizados, encontrándose en $90 \%$ de pacientes satisfechos en el docente, y un $88,6 \%$ en el no docente $(p=0,5)$. Al comparar la variable sexo con satisfacción, se apreció un $81,4 \%$ de mujeres satisfechas contra un $95 \%$ de hombres $(p=0,01)$. Respecto a la variable edad, no hubo diferencia significativa, con una mediana de 50 (43-67) años en no satisfechos y de 63 (51-71) en satisfechos ( $p=0,13)$. Tampoco hubo asociación entre satisfacción y estado civil $(p=0,3)$, nivel educacional $(p=0,46)$, ocupación $(p=0,054)$, ni experiencias previas en otros servicios $(p=0,1)$.

De 70 pacientes encuestados en el centro docente, un $84,4 \%$ respondió "Me agrada" en el ítem "participar en la enseñanza del estudiante de medicina". Al 97,2\% le "Agradaba" o le "Daba igual" ser examinado en presencia de un estudiante, y un $94,3 \%$ sintió que el estudiante respetó su confidencialidad. Un $94,3 \%$ de los pacientes aceptó la presencia del estudiante de Medicina en el centro asistencial (Tabla 3).

\section{DISCUSIÓN}

Los hospitales públicos docente-asistenciales cumplen un rol muy importante en los sistemas de salud, ya que además de permitir el acceso a la salud de los beneficiarios, permiten el entrenamiento de futuros profesionales con un

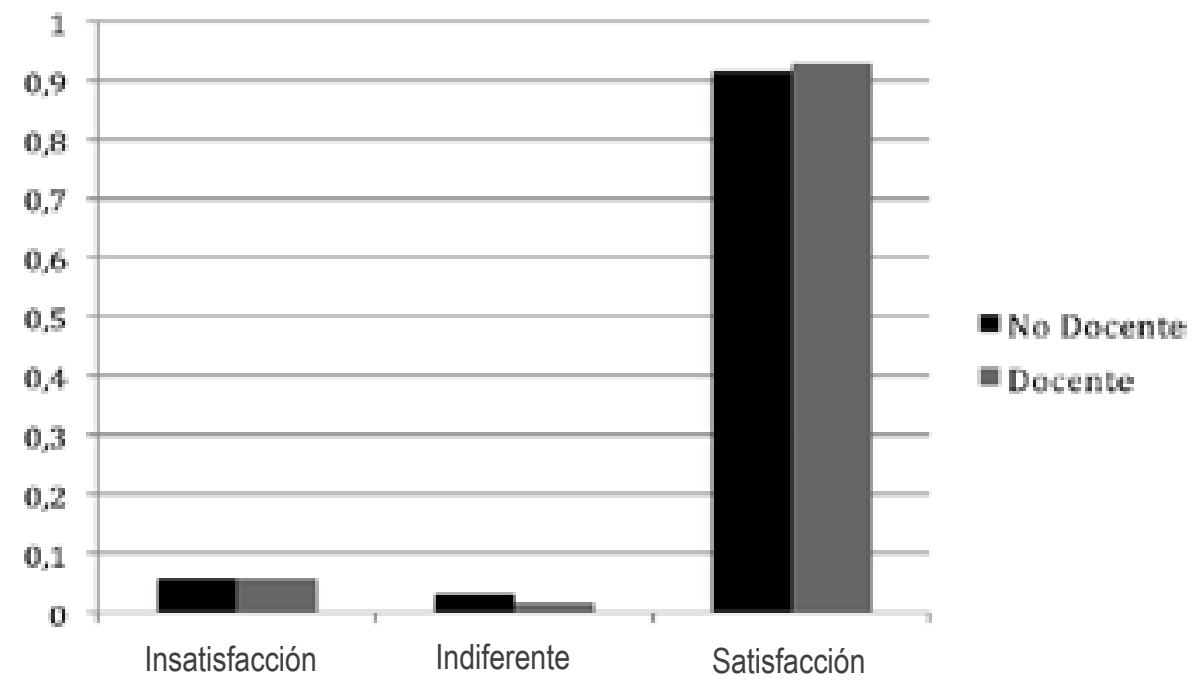

Figura 1. Comparación de la dimensión "Calidad de la atención" entre un servicio docente $(n=70)$ y uno no docente $(n=70)$. 
sentido de misión y trabajo desde la perspectiva del sector público. Incluso para algunos médicos jóvenes el hecho de formarse en hospitales públicos reafirma los valores que los guiaron a estudiar una carrera como medicina, y les entrega innumerables modelos de médicos que han elegido la vida de servicio público ${ }^{25}$. Por lo anterior y en el contexto actual de debate respecto del acceso a una salud más equitativa, es que la formación de futuros médicos adquiere gran relevancia, ya que aún persiste un déficit de cargos en el sistema público. En este sentido es importante resaltar que el beneficio no puede ser solo para los estudiantes y las universidades, sino además para los pacientes y para el sistema. Estos resultados muestran que al menos en esta muestra no se observa un impacto negativo para la presencia de los estudiantes, siendo ampliamente aceptados por los pacientes.

Muchos estudios indican que la presencia del estudiante en el hospital o centro de salud no altera el nivel de satisfacción de los pacientes ${ }^{17}$, $19,20,27,28$ y, en más de uno, los pacientes sienten que el estudiante mejora la calidad de su atención, volviendo deseable su presencia ${ }^{10-12}$. Sin embargo, son pocos los estudios que se han realizado en pacientes hospitalizados, pudiendo citar a dos publicaciones cuyos resultados muestran que el estudiante es considerado por el paciente como un aporte positivo a su salud ${ }^{11,}$ ${ }^{29}$. Los resultados del presente estudio concuerdan con los de la mayor parte de los trabajos, reflejando que la mayoría de los pacientes está de acuerdo con que hayan estudiantes envueltos en el cuidado de su salud.

Hay que destacar la elevada proporción de "Satisfecho" o "Muy satisfecho" en todas las dimensiones evaluadas para ambos centros. Es importante resaltar que la gran mayoría de los pacientes del centro docente presentó una actitud positiva hacia el estudiante, lo que dista de la idea de que este produciría incomodidad en el paciente, afectando su nivel de satisfacción. Respectos a los hallazgos en el centro docente, se observó una gran proporción de respuestas positivas en relación a la presencia del estudiante de Medicina, con un $84,4 \%$ de entrevistados que les agradaba participar en la enseñanza de los estudiantes, un 97,2\% que le agradaba o le daba igual ser examinado en presencia de un estudiante, un $94,3 \%$ que sintió que el estudiante respetó su confidencialidad, y un $94,3 \%$ de entrevistados que aceptaron la presencia de los estudiantes.

La nueva ley acerca de deberes y derechos de las personas en salud aprobada recientemente no puede quedar ajena al análisis. Con ella se resguarda el derecho de todos los pacientes de recibir un trato digno, tener compañía y asistencia espiritual, efectuar consultas y reclamos, ser informados y consentir informadamente, entre otros. En este sentido, se deberá avanzar hacia una cultura de informar a los pacientes cuando un "profesional" que lo atienda sea un alumno, un interno, un residente, o un médico del servicio, y probablemente este tipo de categorías serán más familiares para las personas a futuro. Con esto no sólo se beneficia a los estudiantes en términos del resguardo legal y de la tranquilidad de hacer algo respetando los deseos de los pacientes, sino que -ademásgana el sistema en la medida que los pacientes se involucran y participan más del cuidado de su salud, y de las decisiones pertinentes. De acuerdo a la bibliografía disponible, todo indica que a los pacientes les agrada que el estudiante participe en su cuidado, por lo que no deberían producirse grandes cambios. De todas formas, deben realizarse nuevos estudios a futuro para evaluar el real efecto de la ley sobre este tema, ya que hasta ahora en Chile los pacientes de hospitales públicos no han tenido la posibilidad de opinar abiertamente y rechazar si desean el involucramiento del estudiante en su salud. Todo esto implicará fortalecer el trabajo en conjunto entre centros formadores y hospitales públicos en la enseñanza y desarrollo de principios éticos y habilidades comunicacionales que hagan de los futuros profesionales personas más cercanas, respetuosas, y cálidas con los pacientes.

Las fortalezas de este estudio se centran en haber sido realizado en muestras similares, en servicios de la misma especialidad, y hospitales de igual complejidad, lo que permitiría establecer una adecuada comparación. Además, es un acercamiento a un asunto complejo que debe ser estudiado más detalladamente. Entre sus debilidades, se trata de un muestreo por conveniencia que difícilmente tendrá el poder estadístico esperado, sobre todo si se considera que si 
se busca probar equivalencia se debe plantear una hipótesis de "no inferioridad", lo que aumenta aún más el tamaño muestral necesario. Además, para el análisis de los niveles de satisfacción y grado de aceptación del estudiante, se establecieron puntos de corte arbitrarios para los puntajes del cuestionario. Por último, al haber sido realizado solo en servicios de urología, no se puede asegurar que los resultados puedan replicarse en servicios de otra especialidad o en otras circunstancias.

La principal utilidad de estos resultados es ayudar a aclarar las dudas de estudiantes, docentes y población en general, acerca de la posible incomodidad que produciría la presencia del estudiante de Medicina en pacientes hospitalizados. Esto es relevante ya que estos resultados descartan dicho efecto, en la medida que no hubo diferencia en las dimensiones evaluadas entre ambos centros. Además, en los pacientes que tenían relación con los estudiantes, se apreció una gran aceptación a su presencia $(94,3 \%)$, lo que tiene relación con una evaluación positiva hacia ellos, y debería despejar los cuestionamientos que se pudieran presentar al respecto.

En conclusión, en la muestra estudiada, el estar internado en un centro docente o en uno no docente no afectó la satisfacción de los pacientes con la atención médica. Esto permite plantear que es probable que el estudiante sea más bien un aporte para el bienestar de los pacientes. Todo esto indica que la práctica de docencia en los hospitales es un recurso positivo, y que si es correctamente implementado, permite una formación adecuada de profesionales, además de favorecer los niveles de satisfacción de sus usuarios.

Los autores declaran que este trabajo no contó con financiamiento.

\section{REFERENCIAS}

1. Massip C, Ortiz RM, LLantá MdC, Peña M, Infante I. La evaluación de la satisfacción en salud: un reto a la calidad. Rev Cub Salud Pública. 2008;34(4):1-10.

2. Corbella A, Saturno P. Introducción al concepto y dimensiones de la calidad asistencial: cómo empezar. Madrid: Instituto Nacional de Salud, Secretaría General; 1992. p. 123-9.

3. Strasser S, Davis RM. Measuring patient satisfaction for improved patient services. Ann Arbor, Michigan: Health Administration Press, 1991. Health Administration Press, 1991:210.

4. Bolivar I. Satisfaction with primary medical care: population determinants. Gac Sanit. 1999;13(5):371-83.

5. Carr-Hill RA. The measurement of patient satisfaction. J Public Health Med. 1992;14(3):236-49.

6. Vedsted P, Mainz J, Lauritzen T, Olesen F. Patient and GP agreement on aspects of general practice care. Fam Pract. 2002;19(4):339-43.

7. Castillo L, Dougnac A, Vicente I, Muñoz V, Rojas V. Los predictores de satisfacción de pacientes en un centro hospitalario universitario. Rev Med Chil. 2007;135(6):696-701.

8. Ramos-Rodríguez C. Percepción de las relaciones médico-paciente, por parte de los usuarios externos de un Departamento de medicina. An Fac Med. 2008;69:12-16.

9. Tengilimoglu D, Kisa A, Dziegielewski SF. Patient satisfaction in Turkey: differences between public and private hospitals. J Community Health. 1999 Feb;24(1):73-91..

10. Sousa Ade C, Tajra Cda R, Coelho RDE S, Gomes $\mathrm{CM}$, Teixeira RA. Medical learning in a private hospital: patients' and companions' perspectivas. Sao Paulo Med J. 2009;127(2):101-4.

11. Beca JP, Browne F, Valdevenito C, Bataszew A, Martínez MJ. Relación estudiante-enfermo: visión del paciente. Rev Med Chil. 2006;134(8):955-9.

12. Mol SS, Peelen JH, Kuyvenhoven MM. Patients' views on student participation in general practice consultations: a comprehensive review. Med Teach. 2011;33(7):e397-400.

13. Kuan S, O'Donnell JJ. Medical students in the emergency department: how do patients view participation in clinical teaching? Ir Med J. 2007;100(8):560-1.

14. Kianmehr N, Mofidi M, Yazdanpanah R, Ahmadi MA. Medical student and patient perspectives on bedside teaching. Saudi Med J. 2010;31(5):565-8.

15. Fletcher KE, Rankey DS, Stern DT. Bedside interactions from the other side of the bedrail. J Gen Intern Med. 2005 Jan;20(1):58-61.

16. Monnickendam SM, Vinker S, Zalewski S, Cohen O, Kitai E. Patients' attitudes towards the presence of medical students in family practice consultations. Isr Med Assoc J. 2001:3(12):903-6.

17. Benson J, Quince T, Hibble A, Fanshawe T, Emery J. Impact on patients of expanded, general practice based, student teaching: observational and qualitative study. BMJ. 2005 Jul 9;331(7508):89. .

18. Abdulghani HM, Al-Rukban MO, Ahmad SS. Patient attitudes towards medical students in Riyadh, Saudi Arabia. Educ Health (Abingdon). 2008 Jul;21(2):69.

19. Simon SR, Peters AS, Christiansen CL, Fletcher RH. The effect of medical student teaching on patient satisfaction in a managed care setting. J Gen Intern Med. 
$2000 \mathrm{Jul} ; 15(7): 457-61$.

20. Price R, Spencer J, Walker J. Does the presence of medical students affect quality in general practice consultations? Medical Education. 2008;42(4):374-81.

21. Scheffer C, Edelhäuser F, Tauschel D, Riechmann M, Tekian A. Can final year medical students significantly contribute to patient care? A pilot study about the perception of patients and clinical staff. Medical Teacher. 2010;32(7):552-7.

22. Pallin DJ, Harris R, Johnson $\mathrm{Cl}$, Giraldez E. Is Consent "Informed" When Patients Receive Care from Medical Trainees? Academic Emergency Medicine. 2008;15(12):1304-8.

23. Graber MA, Pierre J, Charlton M. Patient Opinions and Attitudes toward Medical Student Procedures in the Emergency Department. Academic Emergency Medicine. 2003;10(12):1329-33.

24. Sweeney K, Magin P, Pond D. Patient attitudes - training students in general practice. Aust Fam Physician. 2010 Sep;39(9):676-82.
25. Gourevitch MN, Malaspina D, Weitzman M, Goldfrank LR. The public hospital in American medical education. J Urban Health. 2008 Sep;85(5):779-86.

26. Dvoredsky AE, Rosselot E. El hospital docente del siglo XXI. Rev Hosp Clín Univ Chile. 2009;20:296-301.

27. York NL, Da Rosa DA, Markwell SJ, Niehaus AH, Folse R. Patient's attitudes toward the involvement of medical students in their care. Am J Surg. 1995;169:421-3.

28. Frank SH, Stange KC, Langa D, Workings M. Direct observation of community-based ambulatory encounters involving medical students. JAMA. 1997 Sep 3;278(9):712-6.

29. Callaly E, Yusra M, Sreenan S, McCormack P. Is the Irish bedside best? Irish Journal of Medical Science. 2010;179(2):179-82.

Recibido: 13 de octubre de 2011 Aprobado: 7 de marzo de 2012 